\title{
Laparoscopic colorectal surgery for colorectal polyps: single institution experience
}

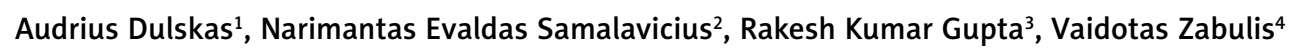 \\ ${ }^{1}$ Department of Abdominal and General Surgery and Oncology, Center of Surgical Oncology, Vilnius, Lithuania \\ ${ }^{2}$ Center of Surgical Oncology, National Cancer Institute, Clinic of Internal Diseases, Family Medicine and Oncology of Medical Faculty, \\ Vilnius, Lithuania \\ ${ }^{3}$ Department of Surgery, Gastrointestinal Unit, B.P. Koirala Institute of Health Sciences, Nepal \\ ${ }^{4}$ Department of Cardiovascular Disease, Center of Reconstructive and Endovascular Surgery, Vilnius, Lithuania
}

Videosurgery Miniinv 2015; 10 (1): 73-78 DOI: $10.5114 /$ wiitm.2015.49752

\begin{abstract}
Introduction: Because of their difficult location or size, some polyps are impossible to remove with a flexible colonoscope and must be surgically removed. Laparoscopy is a great alternative.

Aim: To assess outcomes of a laparoscopic approach for the management of difficult colorectal polyps.

Material and methods: From 2006 to 2014, patients with polyps that could not be treated by endoscopy were included. Demographic data, histology of the biopsy, type of surgery, length of postoperative stay, complications and final pathology were reviewed prospectively.

Results: Forty-two patients with a mean age of $64.9 \pm 8.4$ underwent laparoscopic polypectomy. Laparoscopic mobilization of the colonic segment and colotomy with removal of the polyp was performed for 12 (28.6\%) polyps. Laparoscopic segmental bowel resection was performed in 30 (71.4\%) cases: anterior rectal resection with partial total mesorectal excision in 12 (28.6\%), left hemicolectomy in 7 (16.6\%), sigmoid resection in 6 (14.3\%), ileocecal resection in $2(4.76 \%)$, resection of transverse colon in $2(4.76 \%)$ and sigmoid resection with transanal retrieval of specimen in 1 (2.38\%). Mean postoperative hospital stay was $5.9 \pm 2.6$ days. There were 4 complications (9.5\%). All patients recovered after conservative treatment. Mean polyp size was $3.6 \pm 2.2 \mathrm{~cm}$. Final pathology revealed polyps $(n=2)$, tubular adenoma $(n=6)$, tubulovillous adenoma $(n=20)$, carcinoma in situ $(n=10)$ and invasive cancer $(n=4)$. Two of these patients underwent laparoscopic left hemicolectomies 14 and 10 days after laparoscopic colotomy and polypectomy. Conclusions: For the management of endoscopically unresectable polyps, laparoscopic polypectomy is currently the technique of choice.
\end{abstract}

Key words: laparoscopic surgery, colectomy, colorectal polyp, hand-assisted laparoscopic surgery.

\section{Introduction}

Colorectal cancer (CRC) is the second cause of cancer-related death in the western world [1]. Adenomatous colorectal polyps have a malignant potential well described in Vogelstein's adenoma-carcinoma sequence [2, 3]. Removal of colon adenomas is associated with a reduction in the incidence of CRC.
Thus, CRC largely could be prevented by the detection and removal of adenomatous polyps. There are several methods to remove polyps by using either endoscopic or surgical methods [4]. Patients who have known adenomas and refuse removal develop colon cancer at the rate of $4 \%$ after 5 years and $14 \%$ after 10 years, which is higher than that of the general population [5]. Endoscopic removal of polyps

\section{Address for correspondence}

Audrius Dulskas, Oncology Institute of Vilnius University, 1 Santariskiu St, 08406 Vilnius, Lithuania, phone: +37067520094 ,

e-mail: audrius.dulskas@gmail.com 
has been shown to arrest subsequent development of carcinoma [6].

Because of their difficult location or size, some polyps are impossible to remove using a flexible colonoscope and must be surgically removed. The laparoscopic approach has introduced two technical challenges: firstly the localization of the polyp and secondly its proper surgical extirpation. Depending on the shape of the polyp, its size and location, proper extirpation can be performed through a colotomy (pedunculated or small sessile polyps) or a limited colectomy (large sessile polyps) or an oncological resection in case of suspicion of carcinoma. The potential advantages of laparoscopic surgery are faster recovery, a low rate of incisional hernia, and the reduction of peritoneal adhesions [7].

There have been a number of small reports on laparoscopic removal of colorectal polyps $[7,8]$.

\section{Aim}

In this current study, we report our experience with the laparoscopic removal of 42 'benign' colonic polyps. Based on this patient experience, we more fully describe the broad utility and expected outcomes for laparoscopic removal of colorectal polyps.

\section{Material and methods}

From April 2006 to December 2014, more than 1,000 endoscopic polypectomies were performed at the Department of Surgical Oncology, National Cancer Institute, Vilnius, Lithuania. During the period more than 350 laparoscopic colorectal operations were performed.

A prospectively maintained database was used to identify all patients who underwent laparoscopic polypectomy for polyps that could not be treated by endoscopy due to size, location, and/or risk of complications. Exact reasons why the referring endoscopist could not achieve endoscopic polypectomy were not available. All patients underwent laparoscopic surgery performed by one surgeon (NES). All consenting patients aged 18 years or older with histologically confirmed adenoma were included in this study. Invasive carcinoma was the criterion for exclusion. Demographic data, past surgical history, preoperative polyp morphology and histology of the biopsy, type of surgery, length of postoperative stay, complications, final pathology and stage of cancer (if present) were analyzed prospectively.

\section{Surgical technique}

All laparoscopic procedures were done by one surgeon in the department. Preoperative polyp marking was used if the polyp was not in the cecal area. For marking methylene blue in the morning of the operation was used.

Pre-operative bowel preparation the day before surgery, and intravenous broad-spectrum antibiotics on induction were routine. Under general anesthesia, a $6 \mathrm{~cm}$ umbilical incision was made for the hand-port insertion for hand-assisted laparoscopic surgery (HALS). Trocars were placed according to the type of procedure. If bowel resection was performed, the vascular pedicles were initially isolated by a medial to lateral approach, the ureters identified, and a ligation of the vessels performed. Bowel mobilization was then completed laparoscopically. The specimens were retrieved through transumbilical incision and colotomy, and polypectomy (if the polyp was not circular and possible to remove) or resection and anastomosis performed extra-corporeally. Contraindications for colotomy and polyp removal were: circular polyp and risk of bowel stenosis. For laparoscopic anterior resections intra-corporeal anastomosis with an endoanal circular stapler was done. Hand-assisted laparoscopic surgery was performed for polyps localized in the descending colon, sigmoid and rectum, which was possible for specimen retrieval through transumbilical incision. If straight laparoscopy was done, mobilization of the bowel was performed using the standard technique. The segment was brought through the incision above the mobilized bowel and colotomy, and polypectomy or small resection and anastomosis was done.

Follow-up was performed under our institutional guidelines: colonoscopy annually.

\section{Statistical analysis}

All statistical analyses were performed using software (Statistical Package for the Social Sciences, SPSS Inc., Chicago, IL, USA). All data are presented as mean \pm standard deviation for parametric and median for nonparametric data.

\section{Results}

Patients' demographics are shown in Table I. Patients' body mass index was $27.3 \pm 5.8 \mathrm{~kg} / \mathrm{m}^{2}$ (range: $22-36 \mathrm{~kg} / \mathrm{m}^{2}$ ). Twelve (28.6\%) patients had multiple 
Table I. Demographic data

\begin{tabular}{|c|c|}
\hline Parameter & Results \\
\hline \multicolumn{2}{|l|}{ Gender, $n(\%)$ : } \\
\hline Male & $22(52.4)$ \\
\hline Female & $20(47.6)$ \\
\hline Age, mean $\pm S D$, range [years] & $64.9 \pm 8.4(50-83)$ \\
\hline Previous abdominal surgery, $n$ (\%) & $2(4.8)$ \\
\hline \multicolumn{2}{|l|}{ Preoperative pathology, $n(\%)$ : } \\
\hline Adenoma & $34(81)$ \\
\hline Carcinoma in situ & $8(19)$ \\
\hline $\begin{array}{l}\text { Postoperative hospital stay, } \\
\text { mean } \pm \text { SD, range }[\text { days] }\end{array}$ & $5.9 \pm 2.6(2-14)$ \\
\hline Conversion rate & 0 \\
\hline Mortality rate & 0 \\
\hline
\end{tabular}

polyps (2 or more). Fourteen (33.3\%) patients had comorbidities: 12 of them (28.6\%) cardiac, 2 (4.8\%) diabetes. Laparoscopic mobilization of the colonic segment and colotomy and removal of the polyp were performed for 12 (28.6\%) polyps (Table II). Laparoscopic segmental bowel resection was performed in $30(71.4 \%)$ cases: anterior rectal resection with partial total mesorectal excision in $12(28.6 \%)$, left hemicolectomy in $7(16.6 \%)$, sigmoid resection in $6(14.3 \%)$, ileocecal resection in $2(4.76 \%)$, resection of transverse colon in $2(4.76 \%)$ and sigmoid resection with transanal retrieval of specimen in 1 (2.38\%) (Tables II and III).

Eighteen polyps were in the sigmoid colon (42.8\%), $12(28.6 \%)$ polyps were in the rectum, 3 in the trans-

Table III. Procedures performed

\begin{tabular}{|lc|}
\hline Procedure & Results, $n$ (\%) \\
\hline Colotomy and removal of polyp & $12(28.6)$ \\
\hline Laparoscopic bowel resection: & $30(71.4)$ \\
\hline Rectal resection & $12(28.6)$ \\
\hline Left hemicolectomy & $7(16.6)$ \\
\hline $\begin{array}{l}\text { Ileocecal resection } \\
\text { Resection of transverse colon }\end{array}$ & $6(14.3)$ \\
\hline $\begin{array}{l}\text { Sigmoid resection with transanal } \\
\text { retrieval of specimen }\end{array}$ & $2(4.76)$ \\
\hline
\end{tabular}

Table II. Laparoscopic surgical procedure $(n=42)$

\begin{tabular}{|lc|}
\hline Parameter & Results \\
\hline Right colon: & 2 \\
\hline Colotomy & 2 \\
\hline Ileo-cecal resection & 1 \\
\hline Transverse colon: & 2 \\
\hline Colotomy & 2 \\
\hline Colon resection & 1 \\
\hline Coloncending colon: & 7 \\
\hline Sigmoid and rectum: & 12 \\
\hline Colotomy & 6 \\
\hline Anterior resection & 7 \\
\hline Left hemicolectomy & \\
\hline Sigmoid resection & \\
\hline
\end{tabular}

verse colon (7.1\%) and ascending colon (7.1\%), 2 in the descending colon (4.8\%) and left flexure (4.8\%), and 1 in the right flexure $(2.4 \%)$ and cecum $(2.4 \%)$ (Figure 1).

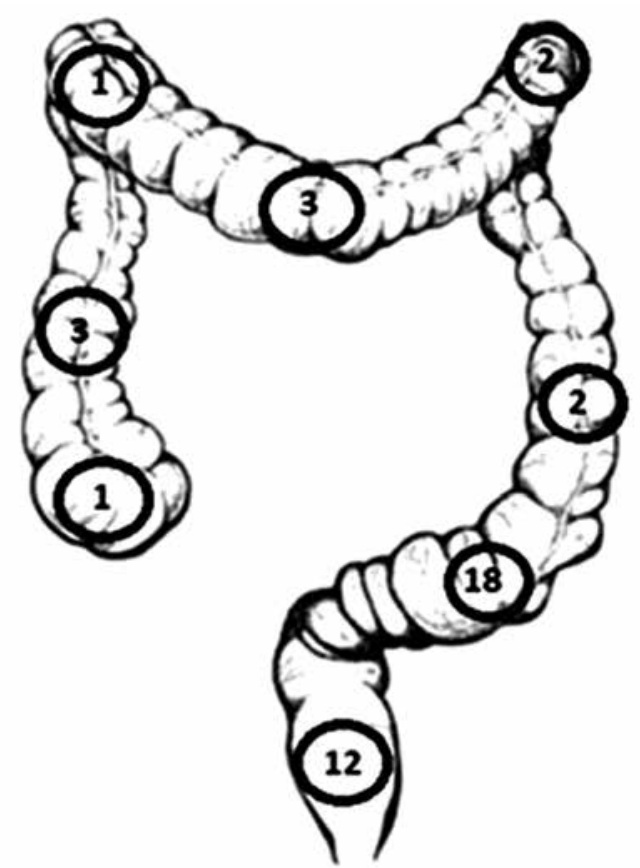

Figure 1. Localization of polyps 
Table IV. Final pathology

\begin{tabular}{|lc|}
\hline Pathology & Results \\
\hline Tubular adenoma & 6 \\
\hline Tubulovillous adenoma & 20 \\
\hline Hyperplastic/juvenile polyp & 2 \\
\hline Carcinoma in situ & 10 \\
\hline Invasive cancer (pT1) & 4 \\
\hline
\end{tabular}

Hand-assisted laparoscopic surgery was performed in 25 patients, straight laparoscopy in others. Mean postoperative hospital stay was $5.9 \pm 2.6$ days (range: 2-14 days). All patients but 4 recovered well and had an uneventful postoperative course. Four postoperative complications were encountered (perioperative morbidity - 9.5\%) - urinary tract infection in 2 patients, partial ileus in 1 and urinary retention in 1 . All patients recovered after conservative treatment. There were no deaths or conversions in our group. Mean polyp size was $3.6 \pm 2.2 \mathrm{~cm}$ (range: 1-10 cm). Final pathology revealed polyps (juvenile and hyperplastic $=2$ ), tubular adenoma $(n=6)$, tubulovillous adenoma $(n=20)$, carcinoma in situ $(n=10)$, pT1 invasive cancer $(n=3)$ and pT2 neuroendocrine cancer $(n=1)$ (Table IV). Invasive carcinoma not identified at colonoscopy and biopsy was found in 4 polyps (9.5\%) - all in tubular adenomas. Two of these patients underwent laparoscopic left hemicolectomies 14 and 10 days after laparoscopic colotomy and polypectomy; the other 2 had oncological bowel resections from the beginning.

All the patients were followed with colonoscopy for 12 months postoperatively, then yearly thereafter. The mean follow-up was 2 years (range: 6 months5 years). There was no incidence of recurrence or any late complications.

Table V. Cancer risk according to polyp size

\begin{tabular}{|lcc|}
\hline Size $[\mathrm{cm}]$ & $\begin{array}{c}\text { Number of polyps } \\
(n=32)\end{array}$ & $\begin{array}{c}\text { Incidence of cancer } \\
n(\%)\end{array}$ \\
\hline$\leq 1$ & 1 & 0 \\
\hline$>1,<2$ & 5 & 0 \\
\hline$\geq 2,<5$ & 26 & $2(7.7)$ \\
\hline$\geq 5$ & 10 & $2(20)$ \\
\hline
\end{tabular}

\section{Discussion}

In the concept of the adenoma-carcinoma sequence, adenomatous polyps are considered to be precursor lesions of colorectal cancer $[2,3]$. The rate of adenomas containing invasive cancer has been estimated between $2.6 \%$ and $9.4 \%$ [7]. Especially, large colonic polyps unresectable at colonoscopy are associated with a high rate of unsuspected cancer. In a study by Pokala et al., postoperative histopathology reports after laparoscopic resection for endoscopically non-resectable polyps revealed adenocarcinomas with an initial benign histology in up to $20 \%$ [8].

Large, sessile polyps or inaccessibility for colonoscopic resection such as the hepatic flexure or the cecum set limits to endoscopy. The size criterion for the definition of 'large' adenomas has varied in the literature. Some authors have called polyps of size $>15 \mathrm{~mm}$ large [6], others > 20-30 mm [9]. Waye suggested the definition of a difficult polyp - a polyp that occupies more than one-third of the bowel circumference or 2 haustral folds [10]. The most common minimum size for an adenoma to be considered 'large' is $20 \mathrm{~mm}$ [9].

In case of endoscopy failure or when malignancy is suspected, a surgical excision should be performed. Surgical options include colotomy in the case of pedunculated polyps and small colectomy in the case of large, broad-base polyps. Polyps that have established or even possible development of malignant transformation require a formal oncologic resection with truncal ligation and lymphadenectomy [7, 11, 12]. In our study we performed 12 (28.6\%) colotomies with mobilization of the colon and polypectomy and 30 (71.4\%) bowel resections. Some authors advise not using colotomy because of the high possibility of exposing the abdominal cavity to cancer cells [13].

Besides a higher risk of complications, a polyp size of larger than $2.5 \mathrm{~cm}$ in diameter was shown to be significantly correlated with malignant transformation in $51 \%$ of the cases in the rectum and $34 \%$ in the colon. Furthermore, a published study showed that polyps larger than $3 \mathrm{~cm}$ could be completely excised in only $67-75 \%$ of cases, thus questioning the endoscopic approach [5].

In our study, large polyp size is the commonest cause, after malignant indications, for colorectal polyps not being amenable to endoscopic treatment alone. The median size of these large polyps was 3.6 $\pm 2.2 \mathrm{~cm}$ (range: $1-10 \mathrm{~cm}$ ). 
It is difficult to reliably predict which patients would have invasive cancer verified in their final pathology. The association between increasing polyp size and the possibility of harboring cancer is well known. This is well shown in our study: 4 of 36 (11.1\%) polyps of size $2 \mathrm{~cm}$ or more (two $2 \mathrm{~cm}$ and two $5 \mathrm{~cm}$ ) harbored invasive cancer (Table V).

Strong emphasis should be placed on preoperative localization of the polyps. Polyps located in the cecal area do not need to be marked. For polyps at any other location, localization procedures are highly recommended: metal clips or injection of dye solutions, such as India ink or methylene blue, can be used for marking $[14,15]$. Polyp marking was used only for 4 patients who underwent straight laparoscopic surgery. We did not use it more often because mostly HALS was performed. Intraoperative colonoscopy may be performed as well; however, it may result in hyperinflation of the bowel, thus obscuring the laparoscopic view [16]. We used intraoperative colonoscopy for 1 patient.

Until recently in our hospital, small resection or anatomical bowel resections were performed by laparotomy, which is associated with a prolonged hospital length of stay and a prolonged postoperative ileus [6]. Laparoscopic colorectal resection has been proven to have faster recovery, decreased morbidity and equivalent long-term oncological safety compared to open procedures $[11,13]$. Our study results, post-operative stay, mortality, conversion rate and complications are comparable to those reported in the literature $[6,7,9,12]$.

The disadvantages of straight laparoscopic procedures are the lack of tactility and the difficulties in defining the extent of the resection [6.] These disadvantages are overcome by HALS or various combinations of laparoscopic-endoscopic "rendezvous" procedures including laparoscopically assisted endoscopic transluminal resection, endoscopically assisted wedge or anatomical resections, and finally an intraoperative tumor location by colonoscopy for achieving oncological resection margins in laparoscopic curative resections. All of these combinations allow a minimally invasive approach for lesions that would otherwise necessitate a laparotomy [7, 8, 17, 18]. Some authors recommend performing a minilaparotomy for colonic polyps [19]. Previous authors reported a $14 \%$ rate of inability to perform splenic flexure mobilization through minilaparotomy [19, 20]. The conversion rate in HALS is estimated from
0 to $10 \%$ [21]. Overall it is easier to perform extended mobilization and resection with HALS compared to minilaparotomy [22]. To perform minilaparotomy in obese patients is very demanding as well. With new hand-port devices it is possible to perform HALS [23].

This study is hindered by lack of control of endoscopists' criteria for unresectability, as well as the small patient number.

The role of laparoscopic colectomy for cancer has been confirmed to be as oncologically appropriate as open colectomy if anatomic principles for oncologic resection are followed $[12,13]$.

\section{Conclusions}

Large colonic polyps unresectable at colonoscopy are associated with a high rate of unsuspected cancer, and these polyps require a formal oncologic colectomy rather than transcolonic polypectomy. Laparoscopic colectomy offers a safe and effective means of eradicating these polyps with the benefits of early postoperative recovery.

\section{Conflict of interest}

The authors declare no conflict of interest.

\section{References}

1. U.S. Cancer Statistics Working Group. United States Cancer Statistics: 1999-2011 Incidence and Mortality Web-based Report. Atlanta (GA): Department of Health and Human Services, Centers for Disease Control and Prevention, and National Cancer Institute 2014.

2. Vogelstein B, Fearon ER, Hamilton SR, et al. Genetic alterations during colorectal-tumor development. N Engl J Med 1988; 319: 525-32.

3. Cho KR, Vogelstein B. Genetic alterations in the adenoma-carcinoma sequence. Cancer 1992; 70: 1727-31.

4. Monkemuller K, Neumann H, Malfertheiner P, Fry LC. Advanced colon polypectomy. Clin Gastroenterol H 2009; 7: 641-52.

5. Wasif N, Etzioni D, Maggard MA, et al. Trends, patterns, and outcomes in the management of malignant colonic polyps in the general population of the United States. Cancer 2011; 117: 931-7.

6. Winter H, Lang RA, Spelsberg FW, et al. Laparoscopic colonoscopic rendezvous procedures for the treatment of polyps and early stage carcinomas of the colon. Int J Colorectal Dis 2007; 22: 1377-81.

7. Hauenschild L, Bader FG, Laubert T, et al. Laparoscopic colorectal resection for benign polyps not suitable for endoscopic polypectomy. Int J Colorectal Dis 2009; 24: 755-9.

8. Pokala N, Delaney CP, Kiran RP, et al. Outcome of laparoscopic colectomy for polyps not suitable for endoscopic resection. Surg Endosc 2007; 21: 400-3. 
9. Lai JH, Ng KH, Ooi BS, et al. Laparoscopic resection for colorectal polyps: a single institution experience. ANZ J Surg 2011; 81: 275-80.

10. Waye JD. How big is too big? Gastrointest Endosc 1996; 43: 256-7.

11. Nassiopoulos K, Pavlidis TE, Menenakos E, et al. Laparoscopic colectomy in the management of large, sessile, transformed colorectal polyps. JSLS 2005; 9: 58-62.

12. Jang JH, Balik E, Kirchoff D, et al. Oncologic colorectal resection, not advanced endoscopic polypectomy, is the best treatment for large dysplastic adenomas. J Gastrointest Surg 2012; 16: 165-72.

13. Loungnarath R, Mutch MG, Birnbaum EH, et al. Laparoscopic colectomy using cancer principles is appropriate for colonoscopically unresectable adenomas of the colon. Dis Colon Rectum 2010; 53: 1017-22.

14. Botoman VA, Pietro M, Thirlby RC. Localization of colonic lesions with endoscopic tattoo. Dis Colon Rectum 1994; 37: 775-6.

15. Wilhelm D, von Delius S, Weber L, et al. Combined laparoscopic-endoscopic resections of colorectal polyps: 10-year experience and follow-up. Surg Endosc 2009; 23: 688-93.

16. Itah R, Greenberg R, Nir S, et al. Laparoscopic surgery for colorectal polyps. JSLS 2009; 13: 555-9.

17. Franklin Jr ME, Leyva-Alvizo A, Abrego-Medina D, et al. Laparoscopically monitored colonoscopic polypectomy: an established form of endoluminal therapy for colorectal polyps. Surg Endosc 2007; 21: 1650-3.

18. Cruz RA, Ragupathi M, Pedraza R, et al. Minimally invasive approaches for the management of "difficult" colonic polyps. Diagn Ther Endosc 2011; 2011: 1-5.

19. Fleshman JW, Fry RD, Birnbaum EH, Kodner IJ. Laparoscopic-assisted and minilaparotomy approaches to colorectal diseases are similar in early outcome. Dis Colon Rectum 1996; 39: 15-22.

20. Nakagoe T, Sawai T, Tsuji T, Ayabe H. Use of minilaparotomy in the treatment of colonic cancer. Br J Surg 2001; 88: 831-6.

21. Aalbers AG, Doeksen A, Van Berge Henegouwen MI, Bemelman WA. Hand-assisted laparoscopic versus open approach in colorectal surgery: a systematic review. Colorectal Dis 2010; 12: 287-95.

22. Tajima T, Mukai M, Yamazaki M, et al. Comparison of handassisted laparoscopic surgery and conventional laparotomy for colorectal cancer: interim results from a single institution. Oncol Lett 2014; 8: 627-32.

23. Ishida H, Nakada H, Yokoyama M, et al. Minilaparotomy approach for colonic cancer. Surg Endosc 2005; 19: 316-20.

Received: 7.12.2014, accepted: 1.02.2015. 